\title{
Identifying accurate metagenome and amplicon software via a meta-analysis of sequence to taxonomy benchmarking studies
}

Paul P Gardner ${ }^{\text {Corresp., }}{ }_{1,2}$ ， Renee J Watson ${ }^{1}$, Xochitl C Morgan ${ }^{3}$, Jenny L Draper ${ }^{4}$ ， Robert D Finn ${ }^{5}$, Sergio E Morales ${ }^{3}$, Matthew B Stott ${ }^{1}$

${ }^{1}$ Biomolecular Interactions Centre, School of Biological Sciences, University of Canterbury, Christchurch, New Zealand

2 Department of Biochemistry, University of Otago, Dunedin, New Zealand

3 Department of Microbiology and Immunology, University of Otago, Dunedin, New Zealand

4 Institute of Environmental Science and Research, Porirua, New Zealand

5 European Molecular Biology Laboratory, European Bioinformatics Institute (EMBL-EBI), Cambridge, UK

Corresponding Author: Paul P Gardner

Email address: paul.gardner@canterbury.ac.nz

Metagenomic and meta-barcode DNA sequencing has rapidly become a widely-used technique for investigating a range of questions, particularly related to health and environmental monitoring. There has also been a proliferation of bioinformatic tools for analysing metagenomic and amplicon datasets, which makes selecting adequate tools a significant challenge. A number of benchmark studies have been undertaken; however, these can present conflicting results. In order to address this issue we have applied a robust Z-score ranking procedure and a network meta-analysis method to identify software tools that are consistently accurate for mapping DNA sequences to taxonomic hierarchies. Based upon these results we have identified some tools and computational strategies that produce robust predictions. 
1 Identifying accurate metagenome and amplicon software via a 2 meta-analysis of sequence to taxonomy benchmarking studies

3

4

5

6

7

9

\author{
Paul P. Gardner ${ }^{1,2}$, Renee J. Watson ${ }^{1}$, Xochitl C. Morgan ${ }^{3}$, Jenny L. Draper ${ }^{4}$, Robert D. Finn ${ }^{5}$,
} Sergio E. Morales ${ }^{3}$, Matthew B. Stott ${ }^{1}$

1. Biomolecular Interactions Centre, School of Biological Sciences, University of Canterbury, Christchurch, New Zealand.

2. Department of Biochemistry, University of Otago, Dunedin, New Zealand.

3. Department of Microbiology and Immunology, University of Otago, Dunedin, New Zealand.

4. Institute of Environmental Science and Research, Porirua, New Zealand.

5. European Molecular Biology Laboratory, European Bioinformatics Institute (EMBL-EBI), Wellcome Trust Genome Campus, Hinxton, Cambridge CB10 1SD, UK

\title{
Abstract
}

Metagenomic and meta-barcode DNA sequencing has rapidly become a widely-used technique for investigating a range of questions, particularly related to health and environmental monitoring. There has also been a proliferation of bioinformatic tools for analysing metagenomic and amplicon datasets, which makes selecting adequate tools a significant challenge. A number of benchmark studies have been undertaken; however, these can present conflicting results. In order to address this issue we have applied a robust Z-score ranking procedure and a network meta-analysis method to identify software tools that are consistently accurate for mapping DNA sequences to taxonomic hierarchies. Based upon these results we have identified some tools and computational strategies that produce robust predictions.

\section{Introduction}

Metagenomics, meta-barcoding and related high-throughput environmental DNA (eDNA) or microbiome sequencing approaches have accelerated the discovery of small and large scale interactions between ecosystems and their biota. Metagenomics is frequently used as a catchall term to cover metagenomic, meta-barcoding and other environmental DNA sequencing methods, which we will also apply apply. The application of these methods has advanced our understanding of microbiomes, disease, ecosystem function, security and food safety (Cho \& Blaser, 2012; Baird \& Hajibabaei, 2012; Bohan et al., 2017). The classification of DNA sequences can be broadly divided into amplicon (barcoding) and genome-wide (metagenome) approaches. The amplicon, or barcoding, -based approaches target genomic marker sequences such as ribosomal RNA genes (Woese, 1987; Woese, Kandler \& Wheelis, 1990; Hugenholtz \& Pace, 1996; Tringe \& Hugenholtz, 2008) (16S, 18S, mitochondrial 12S), RNase P RNA (Brown 
50

51

52

53

54

55

56

57

58

59

60

61

62

63

64

65

66

67

68

69

70

71

72

73

74

75

76

77

78

79

80

81

82

et al., 1996), or internal transcribed spacers (ITS) between ribosomal RNA genes (Schoch et al., 2012). These regions are amplified from extracted DNA by PCR, and the resulting DNA libraries are sequenced. In contrast, genome-wide, or metagenome, -based approaches sequence the entire pool of DNA extracted from a sample with no preferential targeting for particular markers or taxonomic clades. Both approaches have limitations that influence downstream analyses. For example, amplicon target regions may have unusual DNA features (e.g. large insertions or diverged primer annealing sites), and consequently these DNA markers may fail to be amplified by PCR (Brown et al., 2015). While the metagenome-based methods are not vulnerable to primer bias, they may fail to detect genetic signal from low- abundance taxa if the sequencing does not have sufficient depth, or may under-detect sequences with a high $\mathrm{G}+\mathrm{C}$ bias (Tringe et al., 2005; Ross et al., 2013).

High-throughput sequencing (HTS) results can be analysed using a number of different strategies (Supplementary Figure 1) (Thomas, Gilbert \& Meyer, 2012; Sharpton, 2014; Oulas et al., 2015; Quince et al., 2017). The fundamental goal of many of these studies is to assign taxonomy to sequences as specifically as possible, and in some cases to cluster highly-similar sequences into "operational taxonomic units" (OTUs) (Sneath \& Sokal, 1963). For greater accuracy in taxonomic assignment, metagenome and amplicon sequences may be assembled into longer "contigs" using any of the available sequence assembly tools (Olson et al., 2017; Breitwieser, Lu \& Salzberg, 2017). The reference-based methods (also called "targeted gene assembly") make use of conserved sequences to constrain sequence assemblies. These have a number of reported advantages including reducing chimeric sequences, and improving the speed and accuracy of assembly relative to de novo methods (Zhang, Sun \& Cole, 2014; Wang et al., 2015; Huson et al., 2017; Nurk et al., 2017).

Metagenomic sequences are generally mapped to a reference database of sequences labelled with a hierarchical taxonomic classification. The level of divergence, distribution and coverage of mapped taxonomic assignments allows an estimate to be made of where the sequence belongs in the established taxonomy. This is commonly performed using the lowest common ancestor approach (LCA) (Huson et al., 2007). Some tools, however, avoid this computationally-intensive sequence similarity estimation, and instead use alignment-free approaches based upon sequence composition statistics (e.g. nucleotide or k-mer frequencies) to estimate taxonomic relationships (Gregor et al., 2016).

In this study we identified seven published evaluations, of tools that estimate taxonomic origin from DNA sequences (Bazinet \& Cummings, 2012; Peabody et al., 2015; Lindgreen, Adair \& Gardner, 2016; Siegwald et al., 2017; Mclntyre et al., 2017; Sczyrba et al., 2017; Almeida et al., 2018). Of these, four evaluations met our criteria for a neutral comparison study (Boulesteix, Lauer \& Eugster, 2013) (see Supplementary Table 1). These are summarised in Table 1 (Bazinet \& Cummings, 2012; Lindgreen, Adair \& Gardner, 2016; Siegwald et al., 2017; Almeida et al., 2018) and include accuracy estimates for 25 eDNA classification tools. We have used network meta-analysis techniques and non-parametric tests to reconcile variable and sometimes conflicting reports from the different evaluation studies. Our result is a short list of methods that have been consistently reported to produce accurate interpretations of 
83 metagenomics results. This study reports one of the first meta-analyses of neutral comparison

84 studies, fulfilling the requirement for an apex study in the evidence pyramid for benchmarking

85 (Boulesteix, Wilson \& Hapfelmeier, 2017).

86

87

88

89

90

91

92

93

94

95

96

97

98

\section{Overview of environmental DNA classification evaluations}

Independent benchmarking of bioinformatic software provides a valuable resource for determining the relative performance of software tools, particularly for problems with an overabundance of tools. Some established criteria for reliable benchmarks are: 1 . The main focus of the study should be the evaluation and not the introduction of a new method; 2 . The authors should be reasonably neutral (i.e. not involved in the development of methods included in an evaluation); and 3. The test data, evaluation and methods should be selected in a rational way (Boulesteix, Lauer \& Eugster, 2013). Criteria 1 and 2 are straightforward to determine, but criterion 3 is more difficult to evaluate as it includes identifying challenging datasets and appropriate metrics for accurate accuracy reporting (Boulesteix, 2010; Jelizarow et al., 2010; Norel, Rice \& Stolovitzky, 2011). Based upon our literature reviews and citation analyses, we have identified seven published evaluations of eDNA analysis, we have assessed these against the above three principles and four of these studies meet the inclusion criteria (assessed in Supplementary Table 1) (Bazinet \& Cummings, 2012; Lindgreen, Adair \& Gardner, 2016; Siegwald et al., 2017; Almeida et al., 2018). These studies are summarised in Table 1.

In the following sections we discuss issues with collecting trusted datasets, including the selection of positive and negative control data that avoid datasets upon which methods may have been over-trained. We describe measures of accuracy for predictions and describe the characteristics of ideal benchmarks, with examples of published benchmarks that meet these criteria.

\section{<<PLACE TABLE 1 HERE >}

\section{Positive and negative control dataset selection}

The selection of datasets for evaluating software can be a significant challenge due to the need for these to be independent of past training datasets, reliable, well-curated, robust and representative of the large population of all possible datasets (Boulesteix, Wilson \& Hapfelmeier, 2017). Positive control datasets can be divided into two different strategies, namely the in vitro and in silico approaches for generating mock communities.

In vitro methods involve generating microbial consortia in predetermined ratios of microbial strains, extracting the consortium DNA, sequencing and analysing these using standard eDNA pipelines (Jumpstart Consortium Human Microbiome Project Data Generation Working Group, 2012; Singer et al., 2016b). Non-reference sequences can also be included to this mix as a form of negative control. The accuracy of the genome assembly, genome partitioning (binning) and read depth proportional to consortium makeup can then be used to confirm software accuracy. In principle, every eDNA experiment could employ in vitro positive and negative controls by "spiking" known amounts of DNA from known sources, as has been widely used for gene expression analysis (Yang, 2006) and increasingly for eDNA experiments (Bowers et al., 2015; 
127 Singer et al., 2016a; Hardwick et al., 2018).

128 In silico methods use selected publicly-available genome sequences. Simulated metagenome 129 sequences can be derived from these (Richter et al., 2008; Huang et al., 2012; Angly et al., 130 2012; Caboche et al., 2014). It is important to note that ideally-simulated sequences are derived 131 from species that are not present in established reference databases, as this is a more realistic 132 simulation of most eDNA surveys. A number of different strategies have been used to control for 133 this (Peabody et al., 2015; Lindgreen, Adair \& Gardner, 2016; Sczyrba et al., 2017). Peabody et 134 al. used "clade exclusion", in which sequences used for an evaluation are removed from 135 reference databases for each software tool (Peabody et al., 2015). Lindgreen et al. used 136 "simulated evolution" to generate simulated sequences of varying evolutionary distances from 137 reference sequences (Lindgreen, Adair \& Gardner, 2016), similarly Almeida et al. simulated 138 random mutations for $2 \%$ of nucleotides in each sequence (Almeida et al., 2018). Sczyrba et al. 139 restricted their analysis to sequences sampled from recently-deposited genomes, increasing the 140 chance that these are not included in any reference databases (Sczyrba et al., 2017). These 141 strategies are illustrated in Figure 1.

142 Another important consideration is the use of negative controls. These can be randomised 143 sequences (Lindgreen, Adair \& Gardner, 2016), or from sequence not expected to be found in 144 reference databases (Mclntyre et al., 2017). The resulting negative-control sequences can be 145 used to determine false-positive rates for different tools. We have summarised the positive and 146 negative control datasets from various published software evaluations in Table 1, along with other features of different evaluations of DNA classification software.

\section{<<PLACE FIGURE 1 HERE >>}

\section{Metrics used for software benchmarking.}

The metrics used to evaluate software play an important role in determining the fit for different tasks. For example, if a study is particularly interested in identifying rare species in samples, then a method with a high true-positive rate (also called sensitivity or recall) may be preferable. Conversely, for some studies, false positive findings may be particularly detrimental, in which case a good true positive rate may be sacrificed in exchange for a lowering the false positive rate. Some commonly used measures of accuracy, including sensitivity (recall/true positive accuracy), specificity (true negative accuracy) and F-measure (the trade-off between recall and precision) are summarised in Table 2.

The definitions of "true positive", "false positive", "true negative" and "false negative" (TP, FP, $\mathrm{TN}$ and $\mathrm{FN}$ respectively) are also an important consideration. There are two main ways this has been approached, namely per-sequence assignment and per-taxon assignment. Estimates of per-sequence accuracy values can be made by determining whether individual sequences were correctly assigned to a particular taxonomic rank (Peabody et al., 2015; Lindgreen, Adair \& Gardner, 2016; Siegwald et al., 2017). Alternatively, per-taxon accuracies can be determined by comparing reference and predicted taxonomic distributions (Sczyrba et al., 2017). The per-taxon approach may lead to erroneous accuracy estimates as sequences may be incorrectly assigned to included taxa. Cyclic-errors can then cancel, leading to inflated accuracy estimates. However, 
171 Successfully recapturing the frequencies of different taxonomic groups as a measure of community diversity is a major aim for eDNA analysis projects. There have been a variety of approaches for quantifying the accuracy of this information. Pearson's correlation coefficient (Bazinet \& Cummings, 2012), L1-norm (Sczyrba et al., 2017), the sum of absolute log-ratios (Lindgreen, Adair \& Gardner, 2016), the log-modulus (Mclntyre et al., 2017) and the Chao 1 error (Siegwald et al., 2017) have each been used. This lack of consensus has made comparing these results a challenge.

The amount of variation between the published benchmarks, including varying taxonomies, taxonomic levels and whether sequences or taxa were used for evaluations can also impede comparisons between methods and the computation of accuracy metrics. To illustrate this we have summarised the variation of F-measures (a measure of accuracy) between the four benchmarks we are considering in this work (Figure 2).

\section{<<PLACE TABLE 2 HERE>>}

186

187

188

189

190

191

192

193

194

195

196

197

198

199

200

201

202

203

204

205

206

207

208

209

210

211

212

\section{Methods}

Literature search: In order to identify benchmarks of metagenomic and amplicon software methods, an initial list of publications was curated. Further literature searches and trawling of citation databases (chiefly Google Scholar) identified a comprehensive list of seven evaluations (Table 1), in which "F-measures" were either directly reported, or could be computed from supplementary materials. These seven studies were then evaluated against the three principles of benchmarking (Boulesteix, Lauer \& Eugster, 2013), four studies meeting all three principles and were included in the subsequent analyses (see Supplementary Table 1 for details).

A list of published eDNA classification software was curated manually. This made use of a community-driven project led by Jonathan Jacobs (Jacobs). The citation statistics for each software publication were manually collected from Google Scholar (in July 2017). These values were used to generate Figure 2.

Data extraction: Accuracy metrics were collected from published datasets using a mixture of manual collection from supplementary materials and automated harvesting of data from online repositories. For a number of the benchmarks, a number of non-independent accuracy estimates were taken, for example different parameters, reference databases or taxonomic levels were used for the evaluations. We have combined all non-independent accuracy measurements using a median value, leaving a single accuracy measures for each tool and benchmark dataset combination. The data, scripts and results are available from: https://github.com/Gardner-BinfLab/meta-analysis-eDNA-software

Data analysis: Each benchmark manuscript reports one or more F-measures for each software method. Due to the high variance of F-measures between studies (see Figure 2C and Supplementary Figure 3 for a comparison), we renormalised the F-measures using the following 
213 formula:

214

$$
\text { Robust.Z.score }=\frac{x_{i}-\operatorname{median}(X)}{\operatorname{mad}(X)}
$$

215 Where the "mad" function is the median absolute deviation, " $X$ " is a vector containing all the $F$ 216 measures for a publication and " $X_{i}$ " is each F-measure for a particular software tool. Robust Z217 scores can then be combined to provide an overall ranking of methods that is independent of 218 the methodological and data differences between studies (Figure 3). The 95\% confidence 219 intervals for median robust Z-scores shown in Figure 3 were generated using 1,000 bootstrap 220 resamplings from the distribution of values for each method, extreme $(F=\{0,1\})$ values seeded 221 into each $X$ in order to capture the full range of potential F-measures.

222

223

224

Network meta-analysis was used to provide a second method that accounts for differences between studies. We used the "netmeta" and "meta" software packages to perform the analysis.

225

226 As outlined in Chapter 8 of the textbook "Meta-Analysis with R", (Schwarzer, Carpenter \& Rücker, 2015), the metacont function with Hedges' $G$ was used to standardise mean differences and estimate fixed and random effects for each method within each benchmark. The 'netmeta'

228 function was then used to conduct a pairwise meta-analysis of treatments (tools) across studies.

229

230 This is based on a graph-theoretical analysis that has been shown to be equivalent to a frequentists network meta-analysis (Rücker, 2012). The 'forest' function was used on the

231

232

233 resulting values to generate Figure $4 \mathrm{~A}$.

234

235

\section{Review of Results}

We have mined independent estimates of sensitivity, positive predictive values (PPV) and Fmeasures for 25 eDNA classification tools, from three published software evaluations. A matrix showing presence-or-absence of software tools in each publication is illustrated in Figure $3 A$.

237

238 Comparing the list of 25 eDNA classification tools to a publicly available list of eDNA classification tools based upon literature mining and crowd-sourcing, we found that $29 \%(25 / 88)$ of all published tools have been evaluated in the four of seven studies we have identified as neutral comparison studies (details in Supplementary Table 1) (Boulesteix, Lauer \& Eugster, 2013). The unevaluated methods are generally recently published (and therefore have not been evaluated yet) or may no longer be available, functional, or provide results in a suitable format for evaluation (see Figure 2A). Several software tools have been very widely cited (Figure $2 \mathrm{~B}$ ), yet caution must be used when considering citation statistics, as the number of citations is not correlated with accuracy (Figure 2D) (Lindgreen, Adair \& Gardner, 2016; Gardner et al., 2017). For example, the tools that are published early are more likely to be widely cited, or it may be that some articles are not necessarily cited for the software tool. For example, the MEGAN1 manuscript is often cited for one of the first implementations of the lowest-common-ancestor (LCA) algorithm for assigning read-similarities to taxonomy (Huson et 250 al., 2007).

251

252

<<PLACE FIGURE 2 HERE >

254

255

After manually extracting sensitivity, PPV and F-measures (or computing these) from the tables 
256 and/or supplementary materials for each publication (Bazinet \& Cummings, 2012; Lindgreen, 257 Adair \& Gardner, 2016; Siegwald et al., 2017; Almeida et al., 2018), we have considered the 258 within-publication distribution of accuracy measures (see Figure 2C, Supplementary Figure 2 \& 259 Supplementary Figure 3). These figures indicate that each publication has differences in F260 measure distributions. These can be skewed and multimodal, and different measures of 261 centrality and variance. Therefore, a correction needs to be used to account for between262 benchmark variation.

264 Firstly, we use a non-parametric approach for comparing corrected accuracy measures. We 265 converted each F-measure to a "robust Z-score" (see Methods). A median Z-score was 266 computed for each software tool, and used to rank tools. A 95\% confidence interval was also 267 computed for each median Z-score using a bootstrapping procedure. The results are presented 268 in Figure 3B (within-benchmark distributions are shown in Supplementary Figure 3). 
271

272

273

274

275

276

277

278

279

280

281

282

283

284

285

286

287

288

289

290

291

292

293

294

295

296

297

298

299

300

301

302

303

304

305

306

307

308

309

310

311

312

\section{<<PLACE FIGURE 3 HERE >>}

The second approach we have used is a network meta-analysis to compare the different results. This approach is becoming widely used in the medical literature, predominantly as a means to compare estimates of drug efficacy from multiple studies that include different cohorts, sample sizes and experimental designs (Lumley, 2002; Lu \& Ades, 2004; Salanti et al., 2008; Higgins et al., 2012; Greco et al., 2015). This approach can incorporate both direct and indirect effects, and incorporates diverse intersecting sets of evidence. This means that indirect comparisons can be used to rank treatments (or software tool accuracy) even when a direct comparison has not been made.

We have used the "netmeta" software utility (implemented in R) (Rücker et al., 2015) to investigate the relative performance of each of the 25 software tools for which we have data, using the F-measure as a proxy for accuracy. A random-effects model and a rank-based approach were used for assessing the relative accuracy of different software tools. The resulting forest plot is shown in Figure 4A.

The two distinct approaches for comparing the accuracies from diverse software evaluation datasets resulted in remarkably consistent software rankings in this set of results. The Pearson's correlation coefficient between robust Z-scores and network meta-analysis oddsratios is $0.91\left(\mathrm{P}\right.$-value $\left.=4.9 \times 10^{-10}\right)$, see Supplementary Figure 6.

\section{<<PLACE FIGURE 4 HERE >}

\section{Conclusions}

The analysis of environmental sequencing data remains a challenging task despite many years of research and many software tools for assisting with this task. In order to identify accurate tools for addressing this problem a number of benchmarking studies have been published (Bazinet \& Cummings, 2012; Peabody et al., 2015; Lindgreen, Adair \& Gardner, 2016; Siegwald et al., 2017; Mclntyre et al., 2017; Sczyrba et al., 2017; Almeida et al., 2018). However, these studies have not shown a consistent or clearly optimal approach.

We have reviewed and evaluated the existing published benchmarks using a network metaanalysis and a non-parametric approach. These methods have identified a small number of tools that are consistently predicted to perform well. Our aim here is to make non-arbitrary software recommendations that are based upon robust criteria rather than how widely-adopted a tool is or the reputation of software developers, which are common proxies for how accurate a software tool is for eDNA analyses (Gardner et al., 2017).

Based upon this meta-analysis, the k-mer based approaches, CLARK (Ounit et al., 2015), Kraken (Wood \& Salzberg, 2014) and One Codex (Minot, Krumm \& Greenfield, 2015) consistently rank well in both the non-parametric, robust Z-score evaluation and the network meta-analysis. The confidence intervals for both evaluations were comparatively small, so these estimates are likely to be reliable. In particular, the network meta-analysis analysis showed that 
313 these tools are significantly more accurate than the alternatives (i.e. the 95\% confidence 314 intervals exclude the the odds-ratio of 1). Furthermore, these results are largely consistent with 315 a recently published additional benchmark of eDNA analysis tools (Escobar-Zepeda et al., 316 2018).

317

318 There were also a number of widely-used tools, MG-RAST (Wilke et al., 2016), MEGAN (Huson 319 et al., 2016) and QIIME 2 (Bokulich et al., 2018) that are both comparatively user-friendly and 320 have respectable accuracy ( $Z>0$ and narrow confidence intervals, see Figure $3 B$ and 321 Supplementary Figure 5). However, the new QIIME 2 tool has only been evaluated in one 322 benchmark (Almeida et al., 2018), and so this result should be viewed with caution until further independent evaluations are undertaken. Therefore QIIME2 has a large confidence interval on the accuracy estimate based upon robust Z-scores (Figure 3) and ranked below high-performing tools with the network meta-analysis (Figure 4). The tools Genometa (Davenport et al., 2012), GOTTCHA (Freitas et al., 2015), LMAT (Ames et al., 2013), mothur (Schloss et al., 2009) and taxator-tk (Dröge, Gregor \& McHardy, 2015), while not meeting the stringent accuracy thresholds we have used above were also consistently ranked well by both approaches.

The NBC tool (Rosen, Reichenberger \& Rosenfeld, 2011) ranked highly in both the robust Zscore and network analysis, however the confidence intervals on both accuracy estimates were comparably large. Presumably, this was due to its inclusion in a single, early benchmark study (Bazinet \& Cummings, 2012) and exclusion from all subsequent benchmarks. To investigate this further, the authors of this study attempted to run NBC themselves, but found that it failed to run (core dump) on test input data. It is possible that with some debugging, this tool could compare favourably with modern approaches.

339

These results can by no means be considered the definitive answer to how to analyse eDNA datasets since tools will continue to be refined and results are based on broad averages over multiple conditions. Therefore, some tools may be more suited for more specific problems than those assessed in these results (e.g. human gut microbiome). Furthermore, we have not addressed the issue of scale -- i.e., do these tools have sufficient speed to operate on the increasingly large-scale datasets that new sequencing methods are capable of producing?

Our analysis has not identified an underlying cause for inconsistencies between benchmarks. We found a core set of software tools that have been evaluated in most benchmarks. These are CLARK, Kraken, MEGAN, and MetaPhyler, but the relative ranking of these tools differed greatly between some benchmarks. We did find that restricting the included benchmarks to those that satisfy the criteria for a "neutral comparison study" (Boulesteix, Lauer \& Eugster, 2013), improved the consistency of evaluations considerably. This may point to differences in the results obtained by "expert users" (e.g. tool developers) compared and those of "amateur users" (e.g. bioinformaticians or microbiologists).

Finally, the results presented in Supplementary Figure 4 indicate that most eDNA analysis tools have a high positive-predictive value (PPV). This implies that false-positive matches between eDNA sequences and reference databases are not the main source of error for these analyses. However, sensitivity estimates can be low and generally cover a broad range of values. This 
359 that matching divergent eDNA and reference database nucleotide sequences remains a 360 significant research challenge in need of further development.

\section{Acknowledgements}

We are grateful to Jonathan Jacobs (@bioinformer) for maintaining a freely accessible list of metagenome analysis methods. We thank the authors of the benchmark studies used for this work, all of whom were offered an opportunity to comment on this work before it was released. In particular: Adam Bazinet, Christopher E. Mason, Alexa Mclntyre, Fiona Brinkman, Alice McHardy, Alexander Sczyrba, David Koslicki and Léa Siegwald for discussions and feedback on our early results. We are grateful to Anne-Laure Boulesteix for valuable suggestions on how to conduct the analyses. 


\section{References}

374 Abubucker S., Segata N., Goll J., Schubert AM., Izard J., Cantarel BL., Rodriguez-Mueller B.,

375 Zucker J., Thiagarajan M., Henrissat B., White O., Kelley ST., Methé B., Schloss PD.,

376 Gevers D., Mitreva M., Huttenhower C. 2012. Metabolic reconstruction for metagenomic

377 data and its application to the human microbiome. PLoS computational biology

$378 \quad$ 8:e1002358.

379 Almeida A., Mitchell AL., Tarkowska A., Finn RD. 2018. Benchmarking taxonomic assignments

380 based on 16S rRNA gene profiling of the microbiota from commonly sampled

381 environments. GigaScience 7. DOI: 10.1093/gigascience/giy054.

382 Ames SK., Hysom DA., Gardner SN., Lloyd GS., Gokhale MB., Allen JE. 2013. Scalable

383 metagenomic taxonomy classification using a reference genome database. Bioinformatics

$384 \quad 29: 2253-2260$.

385 Angly FE., Willner D., Rohwer F., Hugenholtz P., Tyson GW. 2012. Grinder: a versatile

386 amplicon and shotgun sequence simulator. Nucleic acids research 40:e94.

387 Baird DJ., Hajibabaei M. 2012. Biomonitoring 2.0: a new paradigm in ecosystem assessment

388 made possible by next-generation DNA sequencing. Molecular ecology 21:2039-2044.

389 Bazinet AL., Cummings MP. 2012. A comparative evaluation of sequence classification

390 programs. BMC bioinformatics 13:92.

391 Bohan DA., Vacher C., Tamaddoni-Nezhad A., Raybould A., Dumbrell AJ., Woodward G. 2017.

392 Next-Generation Global Biomonitoring: Large-scale, Automated Reconstruction of

$393 \quad$ Ecological Networks. Trends in ecology \& evolution 32:477-487.

394 Bokulich NA., Kaehler BD., Rideout JR., Dillon M., Bolyen E., Knight R., Huttley GA., Gregory

395 Caporaso J. 2018. Optimizing taxonomic classification of marker-gene amplicon sequences 396 with QIIME 2's q2-feature-classifier plugin. Microbiome 6:90. 
397 Boulesteix A-L. 2010. Over-optimism in bioinformatics research. Bioinformatics 26:437-439.

398 Boulesteix A-L., Lauer S., Eugster MJA. 2013. A plea for neutral comparison studies in $399 \quad$ computational sciences. PloS one 8:e61562.

400 Boulesteix A-L., Wilson R., Hapfelmeier A. 2017. Towards evidence-based computational 401 statistics: lessons from clinical research on the role and design of real-data benchmark $402 \quad$ studies. BMC medical research methodology 17:138.

403 Bowers RM., Clum A., Tice H., Lim J., Singh K., Ciobanu D., Ngan CY., Cheng J-F., Tringe SG., 404 Woyke T. 2015. Impact of library preparation protocols and template quantity on the 405 metagenomic reconstruction of a mock microbial community. BMC genomics 16:856.

406 Brady A., Salzberg S. 2011. PhymmBL expanded: confidence scores, custom databases, 407 parallelization and more. Nature methods 8:367.

408 Breitwieser FP., Lu J., Salzberg SL. 2017. A review of methods and databases for metagenomic 409 classification and assembly. Briefings in bioinformatics. DOI: 10.1093/bib/bbx120.

410 Brown CT., Hug LA., Thomas BC., Sharon I., Castelle CJ., Singh A., Wilkins MJ., Wrighton KC., 411 Williams KH., Banfield JF. 2015. Unusual biology across a group comprising more than $412 \quad 15 \%$ of domain Bacteria. Nature 523:208-211.

413 Brown JW., Nolan JM., Haas ES., Rubio MA., Major F., Pace NR. 1996. Comparative analysis 414 of ribonuclease P RNA using gene sequences from natural microbial populations reveals 415 tertiary structural elements. Proceedings of the National Academy of Sciences of the United $416 \quad$ States of America 93:3001-3006.

417 Caboche S., Audebert C., Lemoine Y., Hot D. 2014. Comparison of mapping algorithms used in 418 high-throughput sequencing: application to Ion Torrent data. BMC genomics 15:264.

419 Callahan BJ., McMurdie PJ., Rosen MJ., Han AW., Johnson AJA., Holmes SP. 2016. DADA2: 420 High-resolution sample inference from Illumina amplicon data. Nature methods 13:581421583.

422 Caporaso JG., Kuczynski J., Stombaugh J., Bittinger K., Bushman FD., Costello EK., Fierer N., 
Peña AG., Goodrich JK., Gordon JI., Huttley GA., Kelley ST., Knights D., Koenig JE., Ley RE., Lozupone CA., McDonald D., Muegge BD., Pirrung M., Reeder J., Sevinsky JR., Turnbaugh PJ., Walters WA., Widmann J., Yatsunenko T., Zaneveld J., Knight R. 2010. QIIME allows analysis of high-throughput community sequencing data. Nature methods

Davenport CF., Neugebauer J., Beckmann N., Friedrich B., Kameri B., Kokott S., Paetow M., Siekmann B., Wieding-Drewes M., Wienhöfer M., Wolf S., Tümmler B., Ahlers V., Sprengel F. 2012. Genometa--a fast and accurate classifier for short metagenomic shotgun reads. PloS one 7:e41224.

Dröge J., Gregor I., McHardy AC. 2015. Taxator-tk: precise taxonomic assignment of metagenomes by fast approximation of evolutionary neighborhoods. Bioinformatics $31: 817-824$.

Escobar-Zepeda A., Godoy-Lozano EE., Raggi L., Segovia L., Merino E., Gutiérrez-Rios RM., Juarez K., Licea-Navarro AF., Pardo-Lopez L., Sanchez-Flores A. 2018. Analysis of sequencing strategies and tools for taxonomic annotation: Defining standards for progressive metagenomics. Scientific reports 8:12034.

Freitas TAK., Li P-E., Scholz MB., Chain PSG. 2015. Accurate read-based metagenome characterization using a hierarchical suite of unique signatures. Nucleic acids research 43:e69.

Gardner PP., Paterson JM., Ghomi FA., Umu SUU., McGimpsey S., Pawlik A. 2017. A metaanalysis of bioinformatics software benchmarks reveals that publication-bias unduly influences software accuracy. bioRxiv:092205. DOI: 10.1101/092205. 
449 Gerlach W., Stoye J. 2011. Taxonomic classification of metagenomic shotgun sequences with $450 \quad$ CARMA3. Nucleic acids research 39:e91.

451 Greco T., Biondi-Zoccai G., Saleh O., Pasin L., Cabrini L., Zangrillo A., Landoni G. 2015. The 452 attractiveness of network meta-analysis: a comprehensive systematic and narrative review. $453 \quad$ Heart, lung and vessels 7:133-142.

454 Gregor I., Dröge J., Schirmer M., Quince C., McHardy AC. 2016. PhyloPythiaS+: a self-training 455 method for the rapid reconstruction of low-ranking taxonomic bins from metagenomes. $456 \quad$ PeerJ 4:e1603.

457 Hardwick SA., Chen WY., Wong T., Kanakamedala BS., Deveson IW., Ongley SE., Santini NS., 458 Marcellin E., Smith MA., Nielsen LK., Lovelock CE., Neilan BA., Mercer TR. 2018. Synthetic microbe communities provide internal reference standards for metagenome sequencing and analysis. Nature communications 9:3096.

Higgins JPT., Jackson D., Barrett JK., Lu G., Ades AE., White IR. 2012. Consistency and inconsistency in network meta-analysis: concepts and models for multi-arm studies. Research synthesis methods 3:98-110.

Huang W., Li L., Myers JR., Marth GT. 2012. ART: a next-generation sequencing read simulator. Bioinformatics 28:593-594.

Hugenholtz P., Pace NR. 1996. Identifying microbial diversity in the natural environment: a molecular phylogenetic approach. Trends in biotechnology 14:190-197.

Huson DH., Auch AF., Qi J., Schuster SC. 2007. MEGAN analysis of metagenomic data. Genome research 17:377-386.

Huson DH., Beier S., Flade I., Górska A., El-Hadidi M., Mitra S., Ruscheweyh H-J., Tappu R. 2016. MEGAN Community Edition - Interactive Exploration and Analysis of Large-Scale 473 Huson DH., Tappu R., Bazinet AL., Xie C., Cummings MP., Nieselt K., Williams R. 2017. Fast and simple protein-alignment-guided assembly of orthologous gene families from 
microbiome sequencing reads. Microbiome 5:11.

476

477

478

479

480

481

482

483

484

485

486

487

488

489

490

491

492

493

494

495

496

497

498

499

500

Jacobs J.Metagenomics - Tools, Methods and Madness. Available at https://goo.gl/2gyNxK (accessed August 21, 2017).

Jelizarow M., Guillemot V., Tenenhaus A., Strimmer K., Boulesteix A-L. 2010. Over-optimism in bioinformatics: an illustration. Bioinformatics 26:1990-1998.

Jumpstart Consortium Human Microbiome Project Data Generation Working Group. 2012. Evaluation of $16 S$ rDNA-based community profiling for human microbiome research. PloS one $7: \mathrm{e} 39315$.

Kelley DR., Liu B., Delcher AL., Pop M., Salzberg SL. 2012. Gene prediction with Glimmer for metagenomic sequences augmented by classification and clustering. Nucleic acids research 40:e9.

Langille MGI., Zaneveld J., Caporaso JG., McDonald D., Knights D., Reyes JA., Clemente JC., Burkepile DE., Vega Thurber RL., Knight R., Beiko RG., Huttenhower C. 2013. Predictive functional profiling of microbial communities using 16S rRNA marker gene sequences. Nature biotechnology 31:814-821.

Lever J., Krzywinski M., Altman N. 2016. Points of Significance: Classification evaluation. Nature methods 13:603-604.

Lindgreen S., Adair KL., Gardner PP. 2016. An evaluation of the accuracy and speed of metagenome analysis tools. Scientific reports 6:19233.

Liu B., Gibbons T., Ghodsi M., Pop M. 2010. MetaPhyler: Taxonomic profiling for metagenomic sequences. In: 2010 IEEE International Conference on Bioinformatics and Biomedicine (BIBM). 95-100.

Liu J., Wang H., Yang H., Zhang Y., Wang J., Zhao F., Qi J. 2013. Composition-based classification of short metagenomic sequences elucidates the landscapes of taxonomic and functional enrichment of microorganisms. Nucleic acids research 41:e3.

Lu G., Ades AE. 2004. Combination of direct and indirect evidence in mixed treatment 
comparisons. Statistics in medicine 23:3105-3124.

502 Lu J., Breitwieser FP., Thielen P., Salzberg SL. 2017. Bracken: estimating species abundance 503 in metagenomics data. PeerJ Computer Science 3:e104.

504 Lumley T. 2002. Network meta-analysis for indirect treatment comparisons. Statistics in $505 \quad$ medicine 21:2313-2324.

506 Mclntyre ABR., Ounit R., Afshinnekoo E., Prill RJ., Hénaff E., Alexander N., Minot SS., Danko 507 D., Foox J., Ahsanuddin S., Tighe S., Hasan NA., Subramanian P., Moffat K., Levy S., 508 Lonardi S., Greenfield N., Colwell RR., Rosen GL., Mason CE. 2017. Comprehensive 509 benchmarking and ensemble approaches for metagenomic classifiers. Genome biology $510 \quad 18: 182$.

511 Minot SS., Krumm N., Greenfield NB. 2015. One codex: a sensitive and accurate data platform 512 for genomic microbial identification. bioRxiv.

513 Norel R., Rice JJ., Stolovitzky G. 2011. The self-assessment trap: can we all be better than 514 average? Molecular systems biology 7:537.

515 Nurk S., Meleshko D., Korobeynikov A., Pevzner PA. 2017. metaSPAdes: a new versatile 516 metagenomic assembler. Genome research 27:824-834.

517 Olson ND., Treangen TJ., Hill CM., Cepeda-Espinoza V., Ghurye J., Koren S., Pop M. 2017. 518 Metagenomic assembly through the lens of validation: recent advances in assessing and 519 improving the quality of genomes assembled from metagenomes. Briefings in 520 bioinformatics. DOI: 10.1093/bib/bbx098.

521 Oulas A., Pavloudi C., Polymenakou P., Pavlopoulos GA., Papanikolaou N., Kotoulas G., 522 Arvanitidis C., lliopoulos I. 2015. Metagenomics: tools and insights for analyzing next523 generation sequencing data derived from biodiversity studies. Bioinformatics and biology $524 \quad$ insights 9:75-88.

525 Ounit R., Wanamaker S., Close TJ., Lonardi S. 2015. CLARK: fast and accurate classification of 526 metagenomic and genomic sequences using discriminative k-mers. BMC genomics 16:236. 
527 Patil KR., Haider P., Pope PB., Turnbaugh PJ., Morrison M., Scheffer T., McHardy AC. 2011.

528 Taxonomic metagenome sequence assignment with structured output models. Nature

$529 \quad$ methods 8:191-192.

530 Peabody MA., Van Rossum T., Lo R., Brinkman FSL. 2015. Evaluation of shotgun

531 metagenomics sequence classification methods using in silico and in vitro simulated

532 communities. BMC bioinformatics 16:363.

533 Quince C., Walker AW., Simpson JT., Loman NJ., Segata N. 2017. Shotgun metagenomics, 534 from sampling to analysis. Nature biotechnology 35:833-844.

535 Rho M., Tang H., Ye Y. 2010. FragGeneScan: predicting genes in short and error-prone reads. $536 \quad$ Nucleic acids research 38:e191.

537 Richter DC., Ott F., Auch AF., Schmid R., Huson DH. 2008. MetaSim: a sequencing simulator $538 \quad$ for genomics and metagenomics. PloS one 3:e3373.

539 Rosen GL., Reichenberger ER., Rosenfeld AM. 2011. NBC: the Naive Bayes Classification tool 540 webserver for taxonomic classification of metagenomic reads. Bioinformatics 27:127-129.

541 Ross MG., Russ C., Costello M., Hollinger A., Lennon NJ., Hegarty R., Nusbaum C., Jaffe DB.

542 2013. Characterizing and measuring bias in sequence data. Genome biology 14:R51.

543 Rücker G. 2012. Network meta-analysis, electrical networks and graph theory. Research $544 \quad$ synthesis methods 3:312-324.

545 Rücker G., Schwarzer G., Krahn U., König J. 2015. netmeta: Network meta-analysis using 546 frequentist methods. R package version 0. 8-0. Available at)(Accessed December 1, 2016).

547 Salanti G., Higgins JPT., Ades AE., loannidis JPA. 2008. Evaluation of networks of randomized $548 \quad$ trials. Statistical methods in medical research 17:279-301.

549 Schloss PD., Westcott SL., Ryabin T., Hall JR., Hartmann M., Hollister EB., Lesniewski RA., 550 Oakley BB., Parks DH., Robinson CJ., Sahl JW., Stres B., Thallinger GG., Van Horn DJ., 551 Weber CF. 2009. Introducing mothur: open-source, platform-independent, community552 supported software for describing and comparing microbial communities. Applied and 
environmental microbiology 75:7537-7541.

554 Schoch CL., Seifert KA., Huhndorf S., Robert V., Spouge JL., Levesque CA., Chen W., Fungal

555 Barcoding Consortium., Fungal Barcoding Consortium Author List. 2012. Nuclear ribosomal

556 internal transcribed spacer (ITS) region as a universal DNA barcode marker for Fungi.

557 Proceedings of the National Academy of Sciences of the United States of America

$558 \quad$ 109:6241-6246.

559 Schwarzer G., Carpenter JR., Rücker G. 2015. Meta-Analysis with R. Springer.

560 Sczyrba A., Hofmann P., Belmann P., Koslicki D., Janssen S., Dröge J., Gregor I., Majda S.,

561 Fiedler J., Dahms E., Bremges A., Fritz A., Garrido-Oter R., Jørgensen TS., Shapiro N.,

562 Blood PD., Gurevich A., Bai Y., Turaev D., DeMaere MZ., Chikhi R., Nagarajan N., Quince

563 C., Meyer F., Balvočiūtè M., Hansen LH., Sørensen SJ., Chia BKH., Denis B., Froula JL.,

564 Wang Z., Egan R., Don Kang D., Cook JJ., Deltel C., Beckstette M., Lemaitre C.,

565 Peterlongo P., Rizk G., Lavenier D., Wu Y-W., Singer SW., Jain C., Strous M., Klingenberg

566 H., Meinicke P., Barton MD., Lingner T., Lin H-H., Liao Y-C., Silva GGZ., Cuevas DA.,

567 Edwards RA., Saha S., Piro VC., Renard BY., Pop M., Klenk H-P., Göker M., Kyrpides NC.,

568 Woyke T., Vorholt JA., Schulze-Lefert P., Rubin EM., Darling AE., Rattei T., McHardy AC.

569 2017. Critical Assessment of Metagenome Interpretation-a benchmark of metagenomics

$570 \quad$ software. Nature methods 14:1063-1071.

571 Seemann T. 2014. Prokka: rapid prokaryotic genome annotation. Bioinformatics . DOI:

$572 \quad$ 10.1093/bioinformatics/btu153.

573 Sharpton TJ. 2014. An introduction to the analysis of shotgun metagenomic data. Frontiers in

574 plant science 5:209.

575 Siegwald L., Touzet H., Lemoine Y., Hot D., Audebert C., Caboche S. 2017. Assessment of

576 Common and Emerging Bioinformatics Pipelines for Targeted Metagenomics. PloS one

$577 \quad$ 12:e0169563.

578 Singer E., Andreopoulos B., Bowers RM., Lee J., Deshpande S., Chiniquy J., Ciobanu D., Klenk 
579

580

581

582

583

584

585

586

587

588

589

590

591

592

593

594

595

596

597

598

599

600

601

602

603

604

H-P., Zane M., Daum C., Clum A., Cheng J-F., Copeland A., Woyke T. 2016a. Next generation sequencing data of a defined microbial mock community. Scientific data 3:160081.

Singer E., Bushnell B., Coleman-Derr D., Bowman B., Bowers RM., Levy A., Gies EA., Cheng JF., Copeland A., Klenk H-P., Hallam SJ., Hugenholtz P., Tringe SG., Woyke T. 2016b. High-resolution phylogenetic microbial community profiling. The ISME journal 10:20202032.

Sneath A., Sokal RR. 1963. Principles of numerical taxonomy. San Francisco and London I 963.

Stoye J., Evers D., Meyer F. 1998. Rose: generating sequence families. Bioinformatics 14:157163.

Stranneheim H., Käller M., Allander T., Andersson B., Arvestad L., Lundeberg J. 2010. Classification of DNA sequences using Bloom filters. Bioinformatics 26:1595-1600.

Thomas T., Gilbert J., Meyer F. 2012. Metagenomics - a guide from sampling to data analysis. Microbial informatics and experimentation 2:3.

Tringe SG., Hugenholtz P. 2008. A renaissance for the pioneering 16S rRNA gene. Current opinion in microbiology 11:442-446.

Tringe SG., von Mering C., Kobayashi A., Salamov AA., Chen K., Chang HW., Podar M., Short JM., Mathur EJ., Detter JC., Bork P., Hugenholtz P., Rubin EM. 2005. Comparative metagenomics of microbial communities. Science 308:554-557.

Truong DT., Franzosa EA., Tickle TL., Scholz M., Weingart G., Pasolli E., Tett A., Huttenhower C., Segata N. 2015. MetaPhIAn2 for enhanced metagenomic taxonomic profiling. Nature methods 12:902-903.

Wang Q., Fish JA., Gilman M., Sun Y., Brown CT., Tiedje JM., Cole JR. 2015. Xander: employing a novel method for efficient gene-targeted metagenomic assembly. Microbiome 3:32.

Wilke A., Bischof J., Gerlach W., Glass E., Harrison T., Keegan KP., Paczian T., Trimble WL., 
605 Bagchi S., Grama A., Chaterji S., Meyer F. 2016. The MG-RAST metagenomics database 606 and portal in 2015. Nucleic acids research 44:D590-4.

607 Woese CR. 1987. Bacterial evolution. Microbiological reviews 51:221-271.

608 Woese CR., Kandler O., Wheelis ML. 1990. Towards a natural system of organisms: proposal 609 for the domains Archaea, Bacteria, and Eucarya. Proceedings of the National Academy of 610 Sciences of the United States of America 87:4576-4579.

611 Wood DE., Salzberg SL. 2014. Kraken: ultrafast metagenomic sequence classification using 612 exact alignments. Genome biology 15:R46.

613 Yang IV. 2006. [4] Use of External Controls in Microarray Experiments. Methods in enzymology $614 \quad 411: 50-63$.

615 Zhang Y., Sun Y., Cole JR. 2014. A scalable and accurate targeted gene assembly tool (SAT-

616 Assembler) for next-generation sequencing data. PLoS computational biology

$617 \quad 10: e 1003737$.

618 Zhu W., Lomsadze A., Borodovsky M. 2010. Ab initio gene identification in metagenomic 619 sequences. Nucleic acids research 38:e132.

620 


\section{Figure Captions}

622

623

624

625

626

627

628

629

630

631

632

633

634

635

636

637

638

639

640

641

642

643

644

645

646

647

648

649

650

651

652

653

654

655

656

657

658

659

660

661

662

663

664

Figure 1: Three different strategies for generating positive control sequencing datasets, i.e. genome/barcoding datasets of known taxonomic placement that are absent from existing reference databases. These are: A "clade exclusion", where positive control sequences are selectively removed from reference databases (Peabody et al., 2015); B "simulated evolution", where models of sequence evolution are used to generate sequences of defined divergence times from any ancestral sequence or location on a phylogenetic tree e.g. (Stoye, Evers \& Meyer, 1998; Dalquen et al., 2012); and C "new genome sequences" are genome sequences that have been deposited in sequence archives prior to the generation of any reference sequence database used by analysis tools (Sczyrba et al., 2017).

Figure 2: A: More than 80 metagenome classification tools have been published in the last 10 years (Jacobs). A fraction of these (29\%) have been independently evaluated. B: The number of citations for each software tool versus the year it was published. Software tools that have been evaluated are coloured and labelled (using colour combinations consistent with evaluation paper(s), see right). Those that have not been evaluated, yet have been cited $>100$ times are labelled in black. C: Box-whisker plots illustrating the distributions of accuracy estimates based upon reported F-measures using values from 4 different evaluation manuscripts (Bazinet \& Cummings, 2012; Lindgreen, Adair \& Gardner, 2016; Siegwald et al., 2017; Almeida et al., 2018). D: The relationship between publication citation counts and the corresponding tool accuracy estimate, as measured by a normalised F-measure (see Methods for details).

Figure 3: A: a matrix indicating eDNA analysis tools in alphabetical order (named on the right axis) versus a published benchmark on the bottom axis. The circle size is proportional to the number of F-measure estimates from each benchmark. B: a ranked list of eDNA classification tools. The median F-measure for each tool is indicated with a thick black vertical line.

Bootstrapping each distribution (seeded with the extremes from the interval) 1000 times, was used to determine a $95 \%$ confidence interval for each median. These are indicated with thin vertical black lines. Each F-measure for each tool is indicated with a coloured point, colour indicates the manuscript where the value was sourced. Coloured vertical lines indicate the median F-measure for each benchmark for each tool.

Figure 4: A. A forest plot of a network analysis, indicating the estimated accuracy range for each tool. The plot shows the relative F-measure with a 95\% confidence interval for each software tool. The tools are sorted based upon relative performance, from high to low. Tools 
665 with significantly higher F-statistics have a 95\% confidence interval that does not cover the null 666 odds-ratio of 1.

667 B. A network representation of the software tools, published evaluations, ranks for each tool 668 and the median F-measure. The edge-widths indicate the rank of a tool within a publication 669 (based upon median, within-publication, rank). The edge-colours indicate the different 670 publications, and node-sizes indicate median F-measure (based upon all publications). An edge 671 is drawn between tools that are ranked consecutively within a publication.

672 


\section{Figure 1 (on next page)}

Three different strategies for generating positive control sequencing datasets

Three different strategies for generating positive control sequencing datasets, i.e. genome/barcoding datasets of known taxonomic placement that are absent from existing reference databases. These are: "clade exclusion", where positive control sequences are selectively removed from reference databases (Peabody et al., 2015) ; "simulated evolution", where models of sequence evolution are used to generate sequences of defined divergence times from any ancestral sequence or location on a phylogenetic tree e.g. (Stoye, Evers \& Meyer, 1998; Dalquen et al., 2012) ; and "new genome sequences" are genome sequences that have been deposited in sequence archives prior to the generation of any reference sequence database used by analysis tools (Sczyrba et al., 2017) . 
A. Colde exclusion

- Positive control genome (sampled)

- Reference database genome Sequences masked from the reference database

" - Simulated sequence evolution

- . New genome sequences

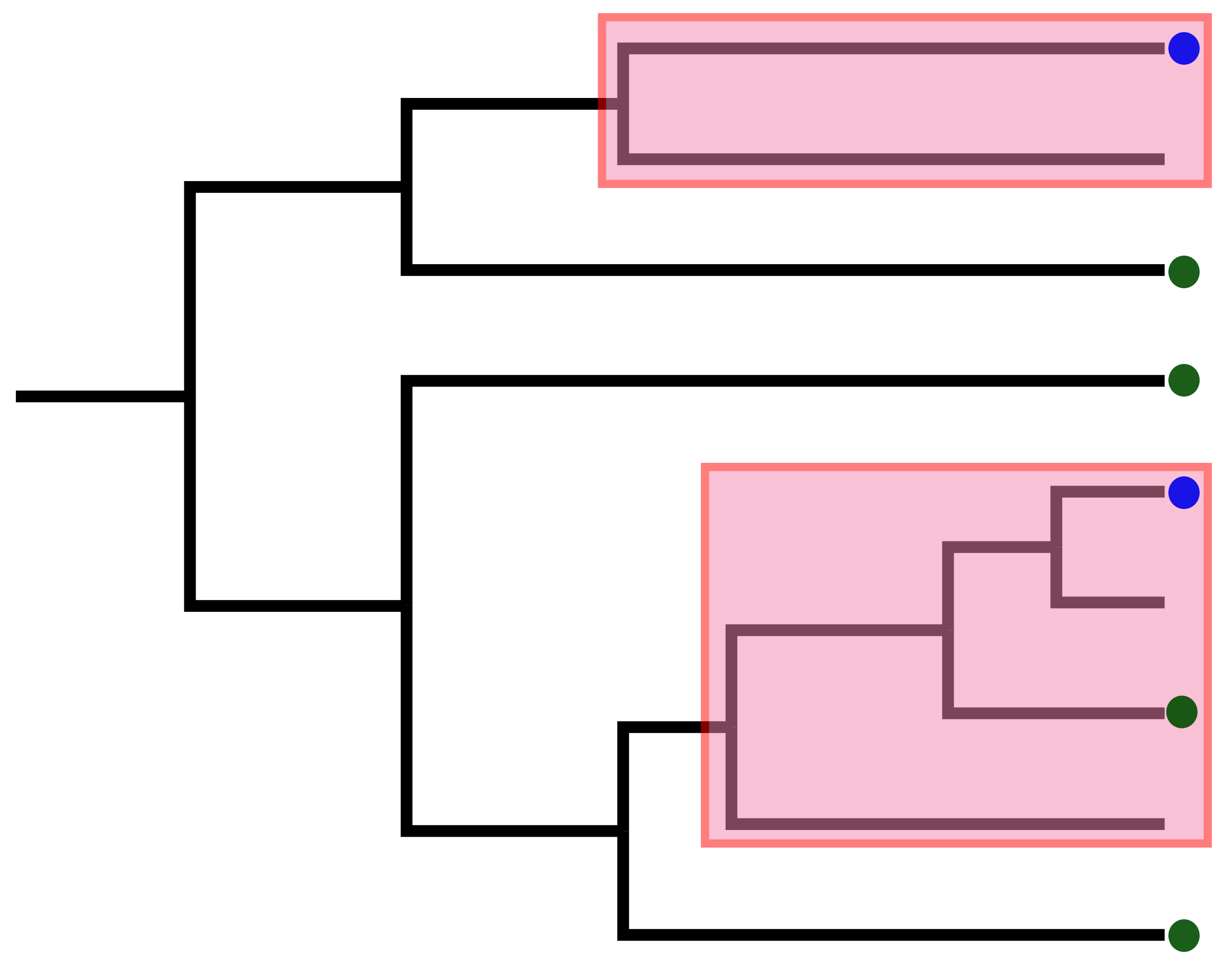

\section{B. Simulated evolution}

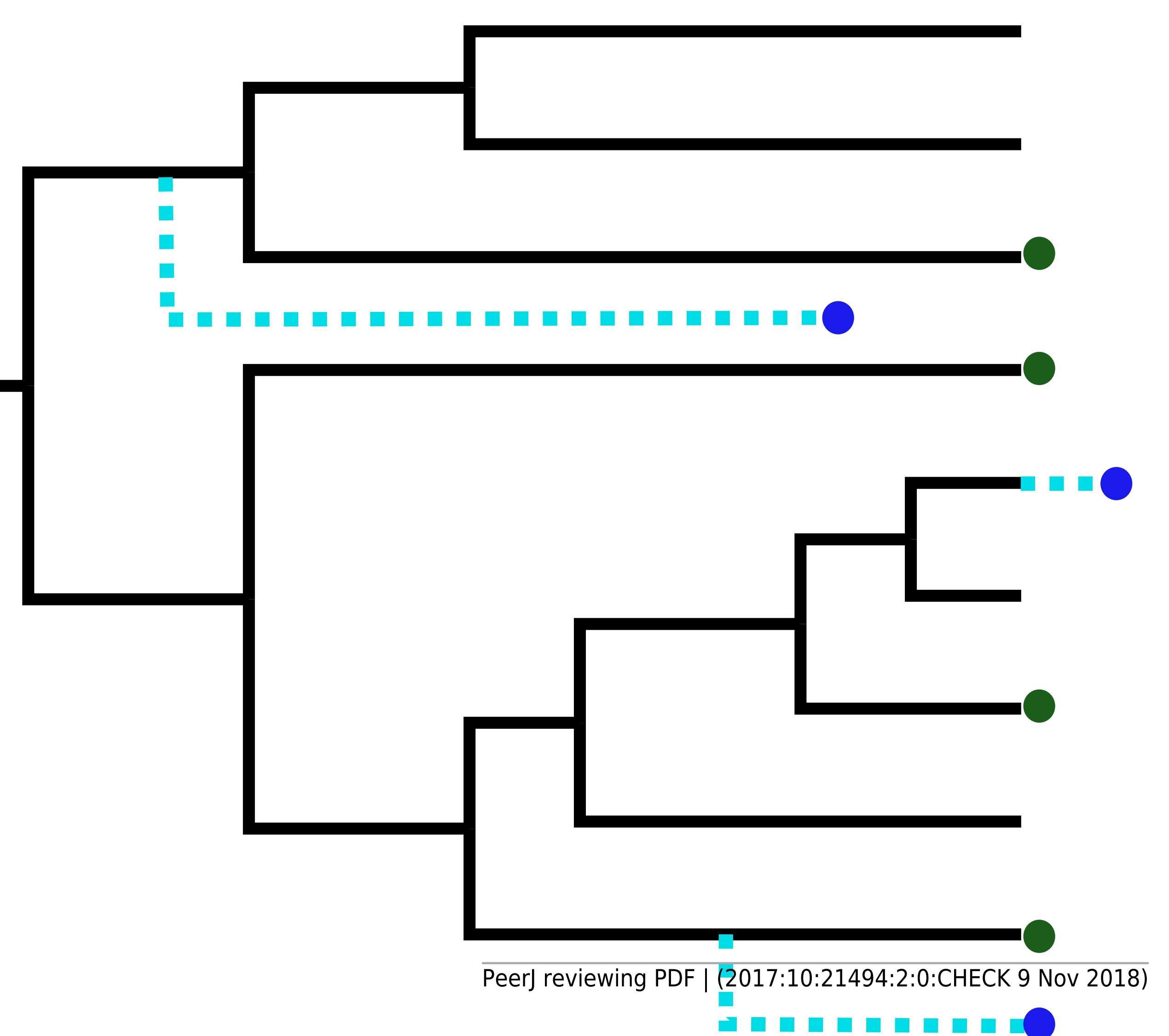

C. New genome sequences

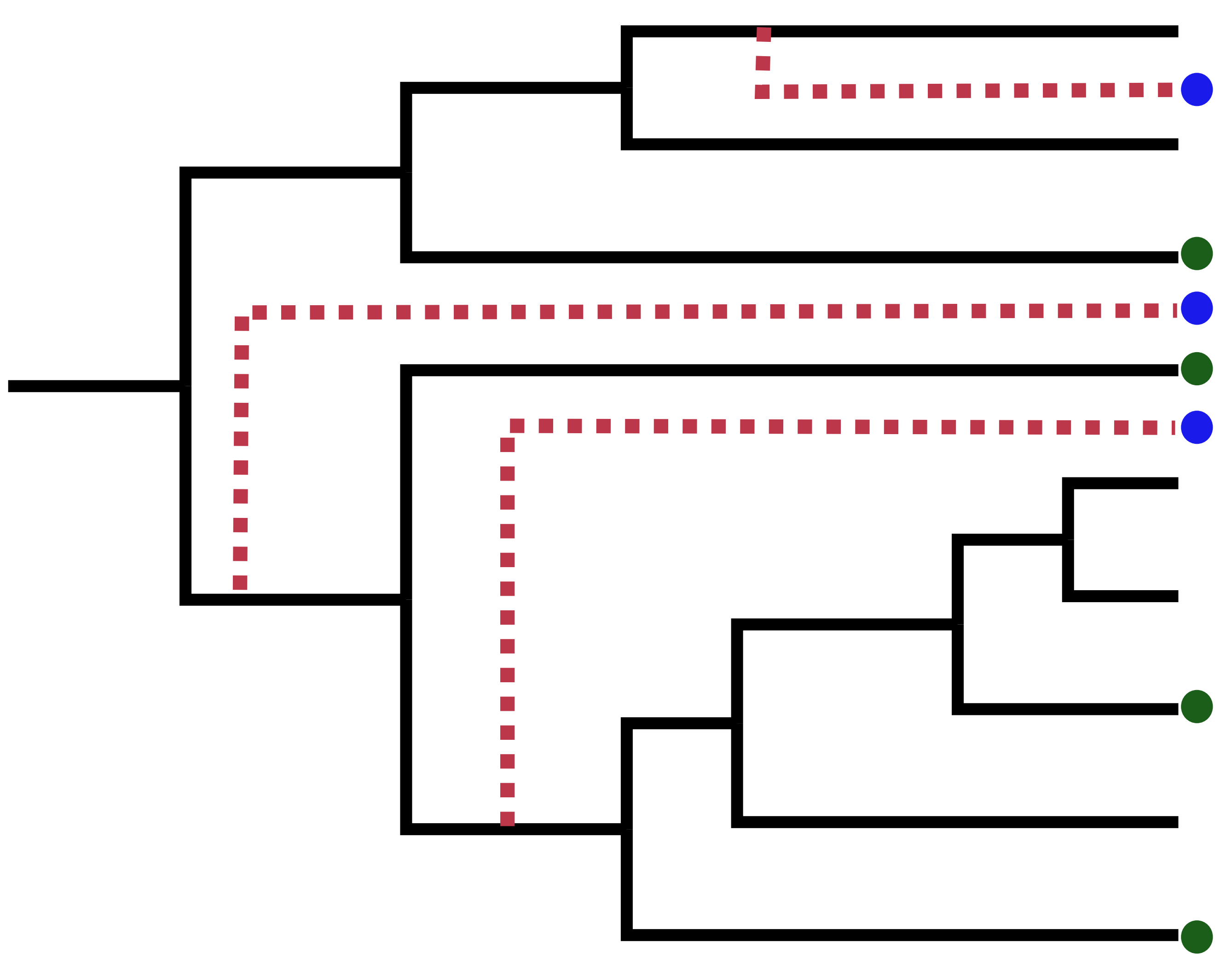

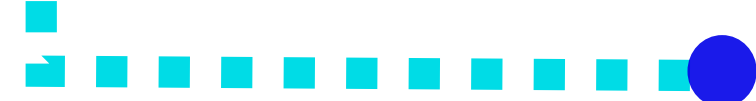




\section{Figure 2 (on next page)}

Summary of software tools, publication dates, citations and benchmarks.

More than 80 metagenome classification tools have been published in the last 10 years (Jacobs) . A fraction of these (29\%) have been independently evaluated. B: The number of citations for each software tool versus the year it was published. Software tools that have been evaluated are coloured and labelled (using colour combinations consistent with evaluation paper(s), see right). Those that have not been evaluated, yet have been cited $>100$ times are labelled in black. C: Box-whisker plots illustrating the distributions of accuracy estimates based upon reported F-measures using values from 4 different evaluation manuscripts (Bazinet \& Cummings, 2012; Lindgreen, Adair \& Gardner, 2016; Siegwald et al., 2017; Almeida et al., 2018) . D: The relationship between publication citation counts and the corresponding tool accuracy estimate, as measured by a normalised F-measure (see Methods for details). 


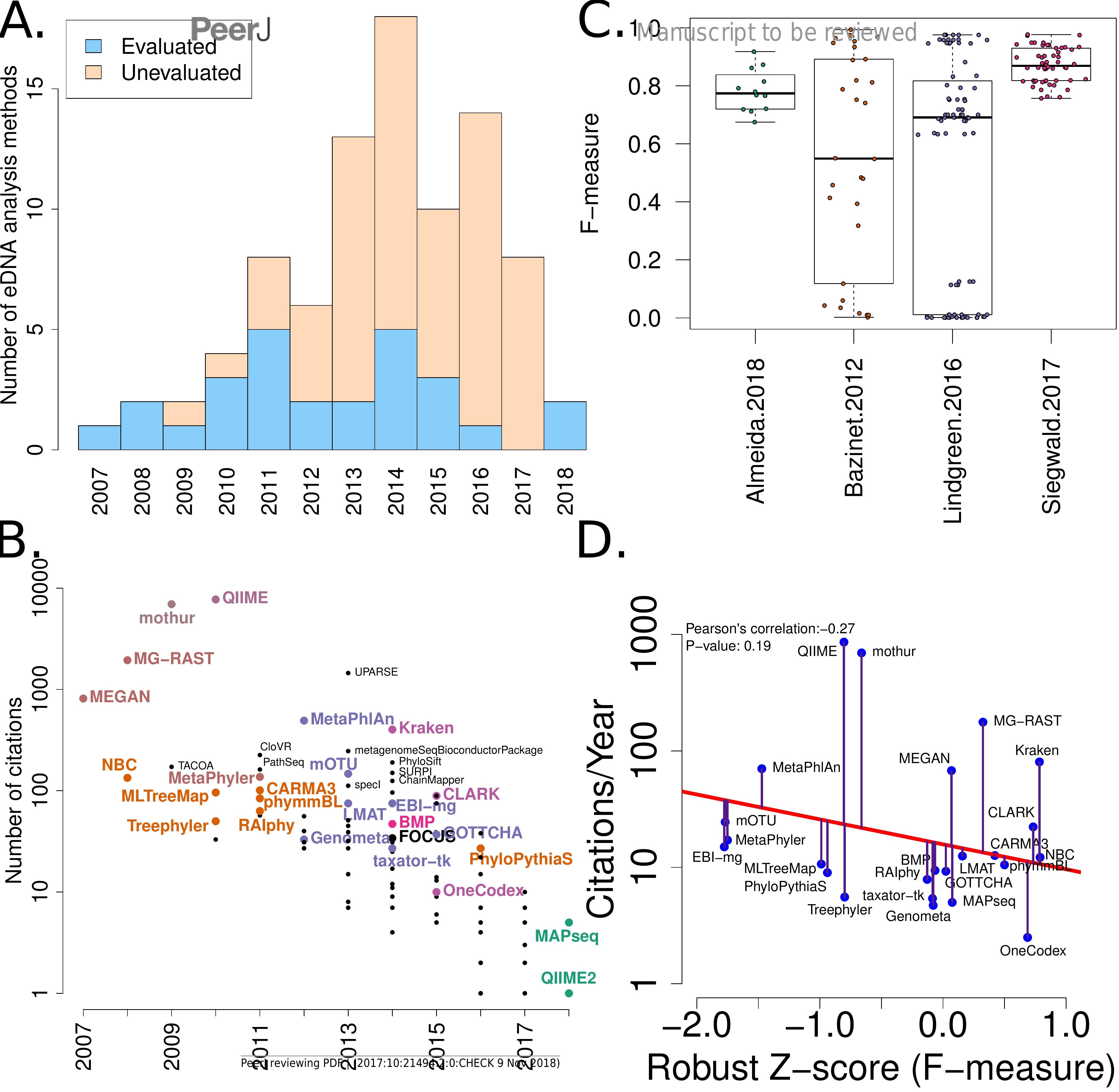

- MACOA hyler: MLTreeMap 8 ${ }^{\circ}$ phymmL Treephyler RÅlphy $\bullet$ MAT BMP taxator-tk PhyloPythias : : :Onéodex : $:$ 


\section{Figure 3 (on next page)}

Robust Z score based comparison of software tools

A: A matrix indicating metagenome analysis tools in alphabetical order (named on the right axis) versus a published benchmark on the bottom axis. The circle size is proportional to the number of F-measure estimates from each benchmark. B: a ranked list of metagenome classification tools. The median F-measure for each tool is indicated with a thick black vertical line. Bootstrapping each distribution (seeded with the extremes from the interval) 1000 times, was used to determine a 95\% confidence interval for each median. These are indicated with thin vertical black lines. Each F-measure for each tool is indicated with a coloured point, colour indicates the manuscript where the value was sourced. Coloured vertical lines indicate the median F-measure for each benchmark for each tool. 


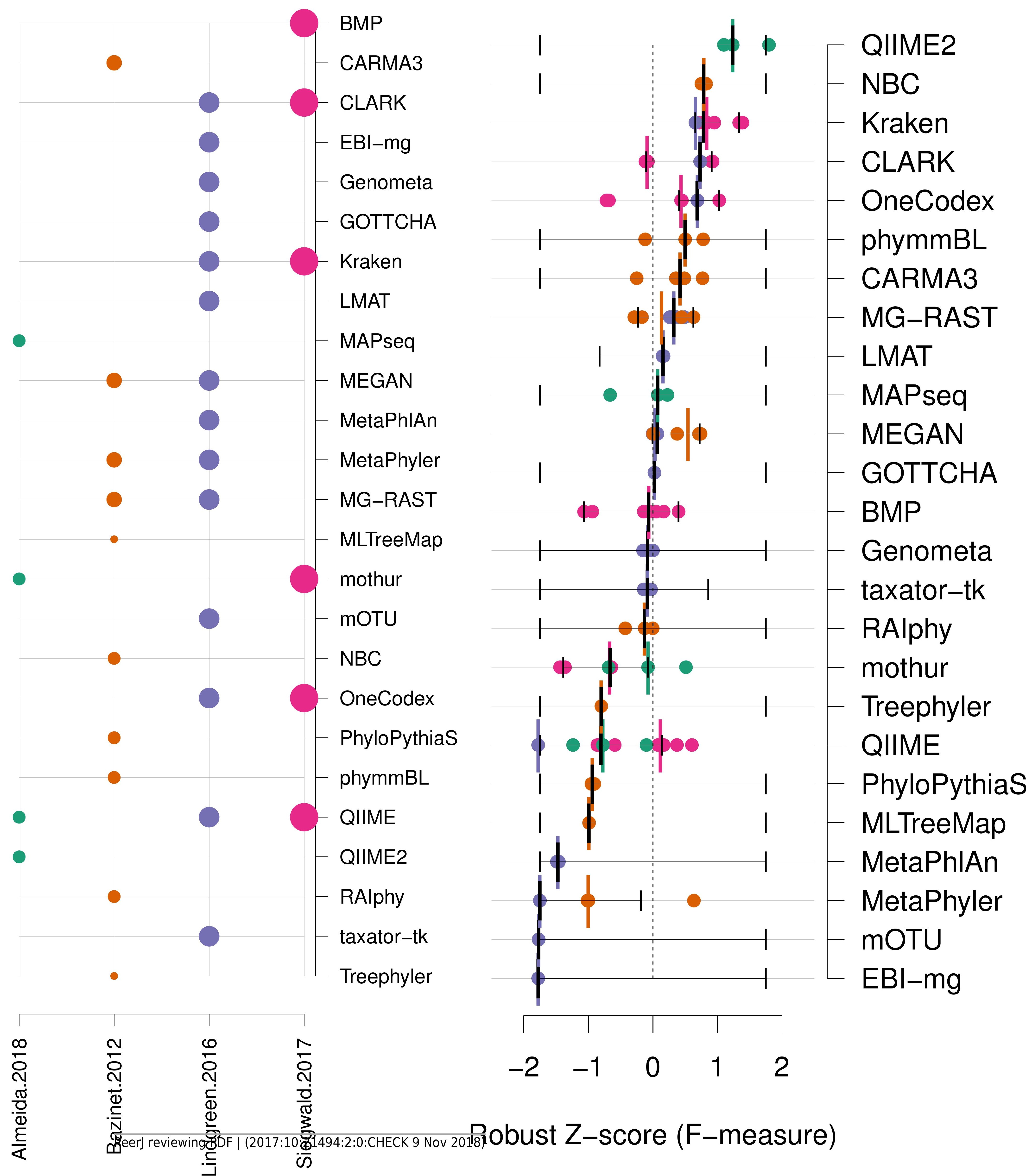




\section{Figure 4 (on next page)}

Network based comparison of metagenome analysis software tools

A. A forest plot of a network analysis, indicating the estimated accuracy range for each tool. The plot shows the relative F-measure with a $95 \%$ confidence interval for each software tool. The tools are sorted based upon relative performance, from high to low. Tools with significantly higher F-statistics have a $95 \%$ confidence interval that does not cover the null odds-ratio of 1. B. A network representation of the software tools, published evaluations, ranks for each tool and the median F-measure. The edge-widths indicate the rank of a tool within a publication (based upon median, within-publication, rank). The edge-colours indicate the different publications, and node-sizes indicate median F-measure (based upon all publications). An edge is drawn between tools that are ranked consecutively within a publication. 


\section{PeerJ}

. Comparison: other vs 'EBI-mg'

Treatment

NBC

Kraken

CLARK

OneCodex

LMAT

MG-RAST

MEGAN

GOTTCHA

CARMA3

phymmBL

Genometa

taxator-tk

BMP

QIIME2

mothur

MAPseq

RAlphy

QIIME

Treephyler

MLTreeMap

MetaPhIAn

PhyloPythias

MetaPhyler

mOTU

$\mathrm{EBI}-\mathrm{mg}$

Prediction interval
(Random Effects Model)

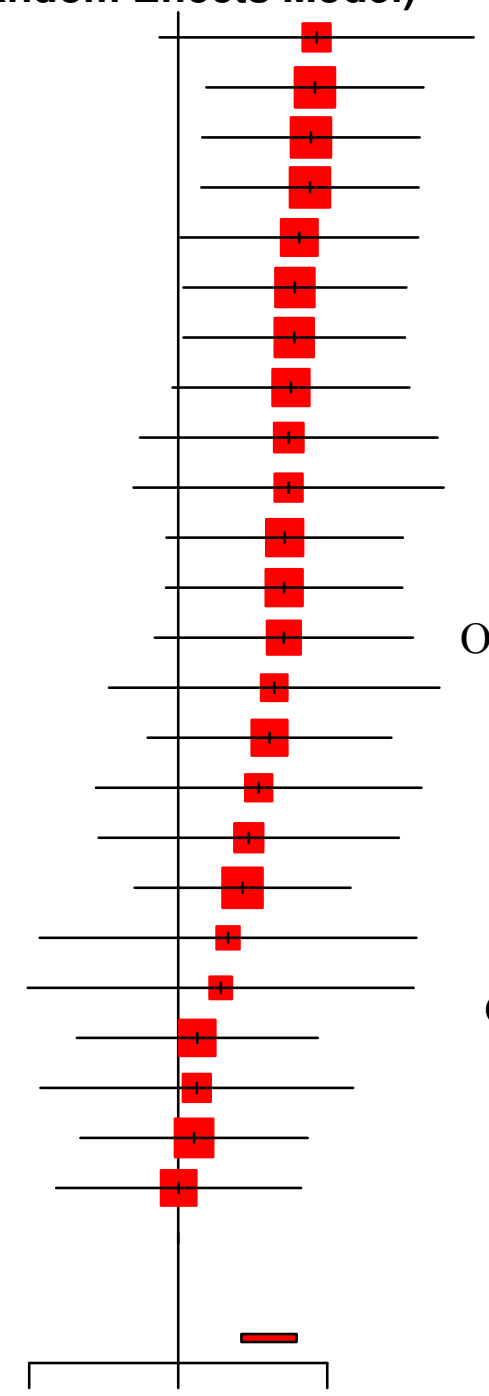

0.5

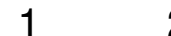

B.

oManus cojat to beoreviewed.6

Median F-measure

QIIME2

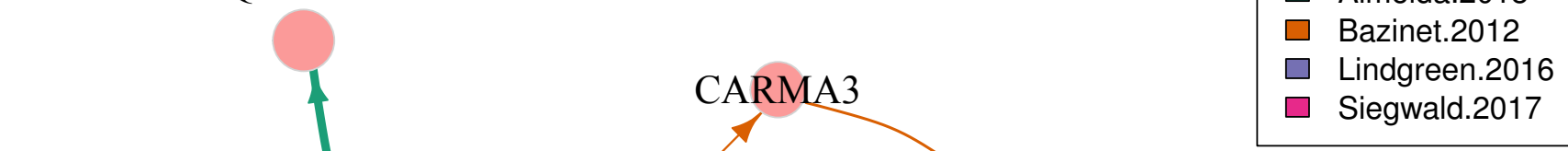

MAPseq

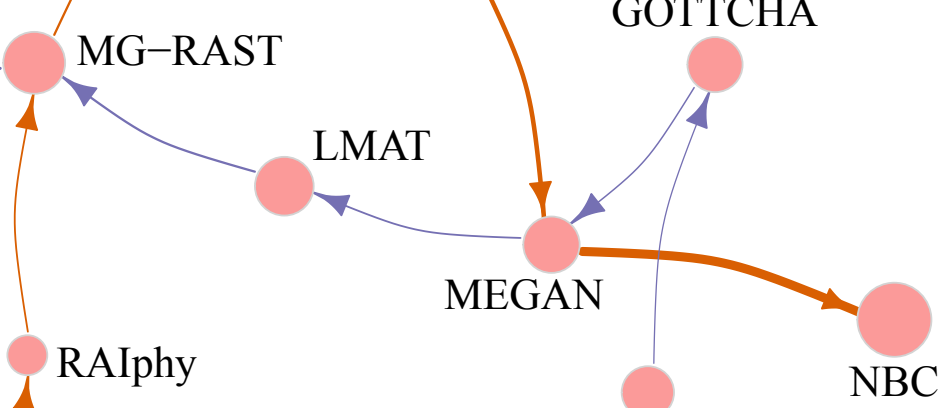

CLARK

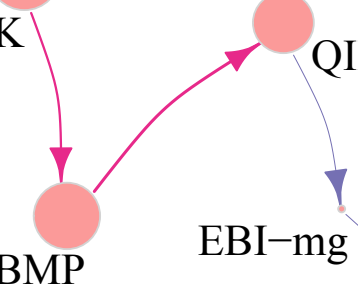

Peer) reviewing PDF | (2017:10:21494:2:0:CHECK 9 Nov 2018)
MG-RAST

phymmBL

Genometa

PhyloPythiaS

Treephyler 4 taxator-tk

MLTreeMap

MetaPhlAn
Almeida.2018

Siegwald 2017 


\section{Table $\mathbf{1}$ (on next page)}

A summary of the main features of the four software evaluations used for this study, including the positive controls employed (the sources of sequences from organisms with known taxonomic placements, whether negative control sequences were used, the approaches for excluding reference sequences from the positive control sequences, and the metrics that were collected for tool evaluation. The accuracy measures are defined in Table 2, the abbreviations used above are Matthews Correlation Coefficient (MCC), Negative Predictive Value (NPV), Positive Predictive Value (PPV), Sensitivity (Sen), Specificity (Spec). 


\begin{tabular}{|c|c|c|c|c|}
\hline Paper & Positive controls & $\begin{array}{l}\text { Negative } \\
\text { controls }\end{array}$ & $\begin{array}{l}\text { Reference } \\
\text { exclusion } \\
\text { method }\end{array}$ & Metrics \\
\hline $\begin{array}{l}\text { Almeida et al. } \\
(2018)\end{array}$ & $\begin{array}{l}12 \text { in silico mock } \\
\text { communities from } 208 \\
\text { different genera. }\end{array}$ & - & $\begin{array}{l}2 \% \text { of positions } \\
\text { "randomly } \\
\text { mutated" }\end{array}$ & $\begin{array}{l}\text { Sequence } \\
\text { Level (Sen., F- } \\
\text { measure) }\end{array}$ \\
\hline $\begin{array}{l}\text { Bazinet et al. } \\
(2012)\end{array}$ & $\begin{array}{l}\text { Four published in silico } \\
\text { mock communities from } \\
742 \text { taxa (Stranneheim et } \\
\text { al., 2010; Liu et al., 2010; } \\
\text { Patil et al., 2011; Gerlach } \\
\text { \& Stoye, 2011) }\end{array}$ & - & - & $\begin{array}{l}\text { Sequence } \\
\text { Level (Sen., } \\
\text { PPV) }\end{array}$ \\
\hline $\begin{array}{l}\text { Lindgreen et al. } \\
(2016)\end{array}$ & $\begin{array}{l}\text { Six in silico mock } \\
\text { communities from } 417 \\
\text { different genera. }\end{array}$ & $\begin{array}{l}\text { Shuffled } \\
\text { sequences }\end{array}$ & $\begin{array}{l}\text { Simulated } \\
\text { evolution }\end{array}$ & $\begin{array}{l}\text { Sequence } \\
\text { Level (Sen., } \\
\text { Spec., PPV, } \\
\text { NPV, MCC) }\end{array}$ \\
\hline $\begin{array}{l}\text { Siegwald et al. } \\
(2017)\end{array}$ & $\begin{array}{ll}36 \text { in silico mock } \\
\text { communities from } 125 \\
\text { bacterial genomes. }\end{array}$ & - & - & $\begin{array}{l}\text { Sequence } \\
\text { Level (Sen, } \\
\text { PPV, } \\
\text { measure) }\end{array}$ \\
\hline
\end{tabular}

3 Table 1: A summary of the main features of the four software evaluations used for this study, 4 including the positive controls employed (the sources of sequences from organisms with known 5 taxonomic placements, whether negative control sequences were used, the approaches for 6 excluding reference sequences from the positive control sequences, and the metrics that were 7 collected for tool evaluation. The accuracy measures are defined in Table 2, the abbreviations 8 used above are Matthews Correlation Coefficient (MCC), Negative Predictive Value (NPV),

9 Positive Predictive Value (PPV), Sensitivity (Sen), Specificity (Spec). 


\section{Table 2 (on next page)}

Some commonly used measures of "accuracy" for software predictions.

These are dependent upon counts of true positives (TP), false positives (FP), true negatives (TN) and false negatives (FN) which can be computed from comparisons between predictions and ground-truths (Lever, Krzywinski \& Altman, 2016) . 


\begin{tabular}{|l|l|l|}
\hline $\begin{array}{l}\text { Sensitivity=TPTP+FN } \\
\text { (a.k.a. recall, true positive rate) }\end{array}$ & $\begin{array}{l}\text { PPV=TPTP+FP } \\
\text { (a.k.a. positive } \\
\text { predictive } \\
\text { value, } \\
\text { precision, } \\
\text { sometimes mis- } \\
\text { labelled } \\
\text { "specificity") }\end{array}$ \\
\hline $\begin{array}{l}\text { F } \\
\text { measure=2*Sensitivity*PPVSensitivity+PPV } \\
=2 T P 2 T P+F P+F N\end{array}$ & Accuracy=TP+TNTP+TN+FP+FN negative rate) & $\begin{array}{l}\text { (a.k.a } \\
\text { positive rate) }\end{array}$ \\
\hline $\begin{array}{l}\text { (a.k.a. F1 score) } \\
\text { false }\end{array}$
\end{tabular}

2

3 Table 2: Some commonly used measures of "accuracy" for software predictions. These are 4 dependent upon counts of true positives (TP), false positives (FP), true negatives (TN) and false 5 negatives (FN) which can be computed from comparisons between predictions and ground6 truths (Lever, Krzywinski \& Altman, 2016). 